\title{
Response: Commentary: "Asking photons where they have been" - without telling them what to say
}

\author{
Ariel Danan, Demitry Farfurnik, Shimshon Bar-Ad and Lev Vaidman * \\ Raymond and Beverly Sackler School of Physics and Astronomy, Tel Aviv University, Tel Aviv, Israel
}

Keywords: quantum information, wave-particle duality, Mach-Zehnder interferometer, weak measurement, photon trajectory

\section{A commentary on}

Commentary: “Asking photons where they have been" - without telling them what to say by Salih H (2015). Front. Phys. 3:47. doi: 10.3389/fphy.2015.00047

In a recent Commentary, Salih [1] claims that we "devised an elegant experiment investigating the past of photons inside two Mach-Zehnder interferometers, one inside the other - yet drew the wrong conclusions [2]." He also argues that the story told by the two-state vector formalism (TSVF) that we advocate, is contradictory. Here we answer Salih's criticism.

Salih considers three possible options for the past of photons in our experiment and argues that

OPEN ACCESS

Edited by: Lorenzo Pavesi,

University of Trento, Italy

Reviewed by:

Yutaka Shikano,

Institute for Molecular Science, Japan

Lorenzo Pavesi,

University of Trento, Italy

*Correspondence: Lev Vaidman vaidman@post.tau.ac.il

Specialty section:

This article was submitted to Optics and Photonics,

a section of the journal

Frontiers in Physics

Received: 18 January 2015 Accepted: 10 June 2015

Published: 30 June 2015

Citation:

Danan A, Farfurnik D, Bar-Ad S and Vaidman L (2015) Response: Commentary: "Asking photons where they have been" - without telling them what to say. Front. Phys. 3:48. doi: 10.3389/fphy.2015.00048 option (1) according to which the photons are present in paths A and B simultaneously, is ruled out. To support his claim he notices that the product of projections on A and on B vanishes. However, for pre- and post-selected systems, as the photons in our experiment, the product rule does not hold [3], and therefore, his argument fails. The photon was in A and in B because it left traces in both places and this is the criterion of the past of the particle we rely on Vaidman [4]. An unavoidable non-vanishing interaction with the environment leads to a "weak measurement" of the presence of the photon in various places inside the interferometer exhibiting "weak-measurement elements of reality" [5].

Our claim, indeed, looks paradoxical. Even if the photon left very small traces in both places, there is a nonvanishing probability that the traces will be identified with certainty. In this case a single photon will be found in two places simultaneously. This is a contradiction: a single photon cannot be detected simultaneously in two places even in a non-demolition measurement. The resolution of the paradox is that the traces in A and in B are entangled and simultaneous detection of the photon in two places cannot happen. The photon changes the reduced density matrix in $\mathrm{A}$ and, also, the reduced density matrix in $\mathrm{B}$, but, if this change is detected in $\mathrm{A}$, then the reduced density matrix in $\mathrm{B}$ becomes identical to the undisturbed density matrix there, and vice versa.

Salih considers a modification of our experiment in which, as he correctly states, we will not observe the presence of the photons in A and in B. However, it is not because the photons were not there, but because the modification spoils the experiment. The weak value of the projection on $\mathrm{A}$ is 1 . This means that the effect on any measuring device weakly coupled to the photon in A will be as if there was a single photon in A. The weak value of the projection on B is - 1 . It does not mean that there is -1 photon, or that the photon has some "negative probability" for being in $\mathrm{B}$. It means that the effect on any measuring device weakly coupled to the photon in B is as if there is one photon in $\mathrm{B}$, but which has a special property of coupling to everything with an opposite sign. The presence of the photon in A shifts the position of the light on the quad-cell detector in one direction while the presence in B equally shifts it in the opposite direction, so the net shift is zero. Salih's modification transforms our experiment to weak measurement of the sum of the projections. 

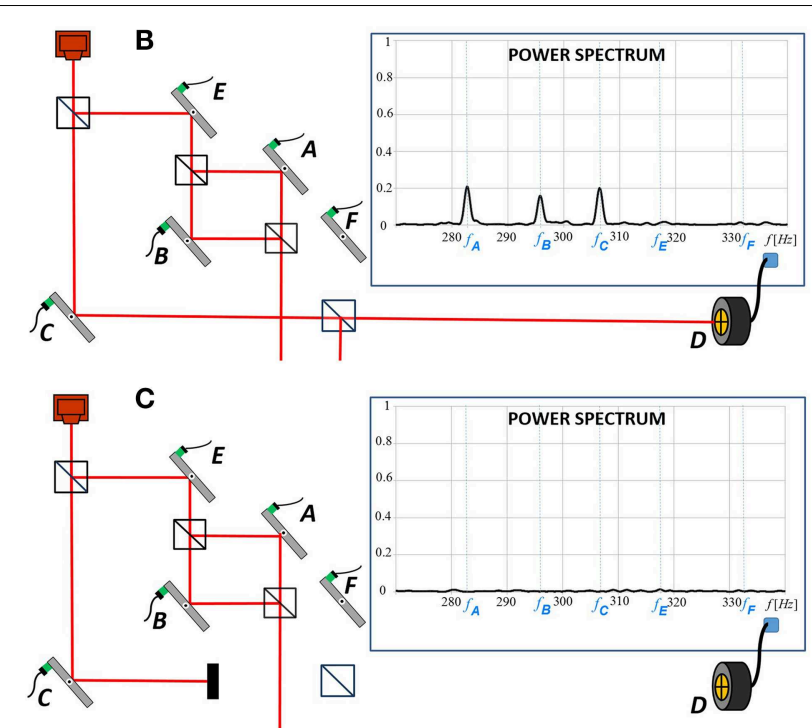

FIGURE 1 | Reproduction of panels (B) and (C) of Figure 2 from [2] When the inner interferometer is tuned in such a way that the beam of light passing through it does not reach the photo-detector, the power spectrum of the signal in the photo-detector still shows frequencies of the mirrors of this interferometer. (C) These frequencies (and all other signals) disappear when we block the lower arm of the large interferometer without changing anything in the upper arm.

The sum rule holds for weak values, so $\left(\mathbf{P}_{A}+\mathbf{P}_{B}\right)_{w}=\left(\mathbf{P}_{A}\right)_{w}+$ $\left(\mathbf{P}_{B}\right)_{w}=0$. Introducing different frequencies of the mirror vibrations in our experiment led to separation of measurements of the projection operators, so we were able to observe the presence of photons in $\mathrm{A}$, in $\mathrm{B}$ (and in $\mathrm{C}$ ).

Salih correctly states that in any experiment in which the amplitude near mirror $\mathrm{F}$ is exactly zero, as in his modification of our experiment, the presence of the photons near A and B will not be detected. This is obvious when we analyze the experiment using solely the forward evolving wave function. As explained in our Letter [2], in this language, it is the small leakage of the wave through $\mathrm{F}$ which is responsible for the effect. But again, the fact that a particular experiment does not show the presence of the photon in $\mathrm{A}$ and $\mathrm{B}$ does not prove that the particle was not there.

Even if one performs local weak measurements of the presence of photons in $\mathrm{A}$ and in $\mathrm{B}$ with external measuring devices, the entanglement with these devices will spoil exact destructive interference and there will be some leakage of the amplitude towards $\mathrm{F}$ (and leakage of the amplitude of the backward evolving state towards $\mathrm{E}$ ). The traces in $\mathrm{F}$ and $\mathrm{E}$ vanish only in the

\section{References}

1. Salih H. Asking photons where they have been without telling them what to say. Front Phys. 3:47. doi: 10.3389/fphy.2015.00047

2. Danan A, Farfurnik D, Bar-Ad S, Vaidman L. Asking photons where they have been. Phys Rev Lett. (2013) 111:240402. doi: 10.1103/PhysRevLett.111.240402 limit of an ideal system without intermediate interactions or measurements. We state that the photons were present in A and $\mathrm{B}$, but not in $\mathrm{E}$ and $\mathrm{F}$ because the ratio between the magnitudes of the traces goes to zero in the limit of weak identical couplings in all places. Thus, in a weak measurement experiment only the traces in A, B (and C) will be observed, as we have seen in our experiment. Note, however, that the presence in $\mathrm{E}$ and $\mathrm{F}$ has a different status relative to places outside the interferometer, so it might be helpful to define a "secondary presence" of the photon in these places [6].

Salih mentions that we have not provided the TSVF analysis of the experiment shown on our Figure 2C ([2]; reproduced here as Figure 1), the case when the channel $C$ was blocked. Indeed, we brought this case not as a demonstration of the power of the TSVF, but for showing that our experimental results in Figure 1B were not due to some technical error of not properly screening electronic signals. Yes, for Figure 1C the simple TSVF analysis fails because the weak values become singular. Since the post-selected state is orthogonal to the forward evolving state, formally, such post-selection is impossible, but the corresponding outcome of the final measurement might happen due to imperfect optical elements and disturbances. The TSVF analysis of this situation is less elegant, but possible, see [7, 8], and it does provide a way to calculate the size of the peaks which were too small to observe in our experiment.

In summary, Salih is correct that in his modification of the experiment the presence of photons in the inner interferometer will not be detected. We argue, however, that the photons are there, Salih's experiment is just not a proper way to observe them. Any experiment which leaves traces in A and B (including ours) leaves some tiny traces in E and F. Salih's suggestion that "the proper" experiment must have exact zero amplitude in F does not correspond to a real world in which there is always some local interaction which leads to nonvanshing (albeit sometimes very small) amplitudes in all paths of the interferometer.

The axioms of standard quantum mechanics do not tell us "where is the particle." Vaidman's proposal [4] to define the past of a photon as places where it leaves a weak trace is consistent and it has been demonstrated in our experiment, including the surprising result of the presence of the trace inside the inner interferometer without observable traces in the paths leading toward or away from it.

\section{Acknowledgments}

This work has been supported in part by the Israel Science Foundation Grant No. 1311/14 and the German-Israeli Foundation Grant No. I-1275-303.14. 
5. Vaidman L. Weak-measurement elements of reality. Found Phys.(1996) 26:895-906. doi: 10.1007/BF02148832

6. Vaidman L. Tracing the past of a quantum particle. Phys Rev A (2014) 89:024102. doi: 10.1103/PhysRevA.89.024102

7. Duck IM, Stevenson PM, Sudarshan ECG. The sense in which a "weak measurement" of a spin- particle's spin component yields a value 100. Phys Rev D (1989) 40:2112. doi: 10.1103/PhysRevD.40.2112

8. Pang S, Wu S, Chen Z-B. Weak measurement with orthogonal preselection and postselection. Phys Rev A (2012) 86:022112. doi: 10.1103/PhysRevA.86. 022112
Conflict of Interest Statement: The authors declare that the research was conducted in the absence of any commercial or financial relationships that could be construed as a potential conflict of interest.

Copyright (c) 2015 Danan, Farfurnik, Bar-Ad and Vaidman. This is an open-access article distributed under the terms of the Creative Commons Attribution License (CC $B Y)$. The use, distribution or reproduction in other forums is permitted, provided the original author(s) or licensor are credited and that the original publication in this journal is cited, in accordance with accepted academic practice. No use, distribution or reproduction is permitted which does not comply with these terms. 